\title{
Billion Colony Forming Units per Gram
}

National Cancer Institute

\section{Source}

National Cancer Institute. Billion Colony Forming Units per Gram. NCI Thesaurus. Code C68901.

A derived unit of viable cell concentration defined as the number of billion colony forming units (10E9 CFU) in one gram of substance. 